\title{
Petroclival Meningioma
}

\author{
Ossama Al-Mefty, M.D. ${ }^{1}$ \\ Laligam N. Sekhar, M.D. ${ }^{2}$ \\ Chandranath Sen, M.D. ${ }^{3}$ \\ Harry R. van Loveren, M.D. ${ }^{4}$
}

\section{CASE HISTORY}

A 28-year-old Hispanic woman complained that she had been experiencing vertigo, ataxia, distal left paresthesias, and left-sided hearing loss for 1 year. Her symptoms had worsened in the last month. On physical examination, she was alert and oriented. Her speech was clear but hesitant. With the exception of left-sided deafness, her cranial nerves were intact. She had a left pronator drift. She had a mild left upper extremity weakness, but her right upper extremity and bilateral lower extremities were normal. The entire left side of her body was hypesthetic. She had mild left dysdiadochokinesia and dysmetria with an ataxic gait. Her toes were upgoing bilaterally. Magnetic resonance imaging (MRI) showed a massive petroclival meningioma extending along the clivus and above and below the tentorium eccentric to the left (Fig. 1).

\section{RESPONSES}

Both the management and surgical treatment of this case are straightforward. This young patient has a large sphenopetroclival meningioma compressing the brain stem and becoming progressively symptomatic. Surgical removal is the pre- ferred treatment, given the size of the tumor and the patient's age and symptoms.

\section{SURGICAL MANAGEMENT}

Intraoperative monitoring is extremely helpful and needs to be extensive. In such a case, we would routinely monitor somatosensory evoked potentials and brain stem auditory evoked responses on the contralateral side to monitor the integrity of the brain stem. The functions of cranial nerves $(\mathrm{CN})$ III, IV, V, VI, VII, IX, X, and XI are monitored by electromyographic recordings from the appropriate muscles innervated by these nerves.

Because hearing is already lost on the left side, the full temporal bone should be used for exposure; that is, a total petrosectomy is needed. Our technique would add the presigmoid petrosal approach, removing the labyrinthine, cochlea, and petrous apex. However, we maintain the skeletonized facial nerve in a small protective bony canal, and we do not transpose the facial nerve to avoid the associated risk of postoperative facial nerve palsy. By itself, using such an approach is paramount in the ability to remove this lesion safely. This approach sacrifices no additional function because hearing is already lost. Typically, both basilar and carotid arteries are involved. Despite the encasement of these vessels, an intervening

${ }^{1}$ Department of Neurosurgery, College of Medicine, University of Arkansas for Medical Sciences, Little Rock, Arkansas; ${ }^{2}$ Mid-Atlantic Brain and Spine Institutes, Annandale, Virginia; ${ }^{3}$ Department of Neurosurgery, St. Luke's-Roosevelt Hospital Center, New York, New York; ${ }^{4}$ Mayfield Clinic, Cincinnati, Ohio. Copyright (C) 2001 by Thieme Medical Publishers, Inc., 333 Seventh Avenue, New York, NY 10001, USA. Tel: +1(212) 584-4662. 1531-5010,p;2001,11,02,143,148,ftx,en;sbs00213x. 

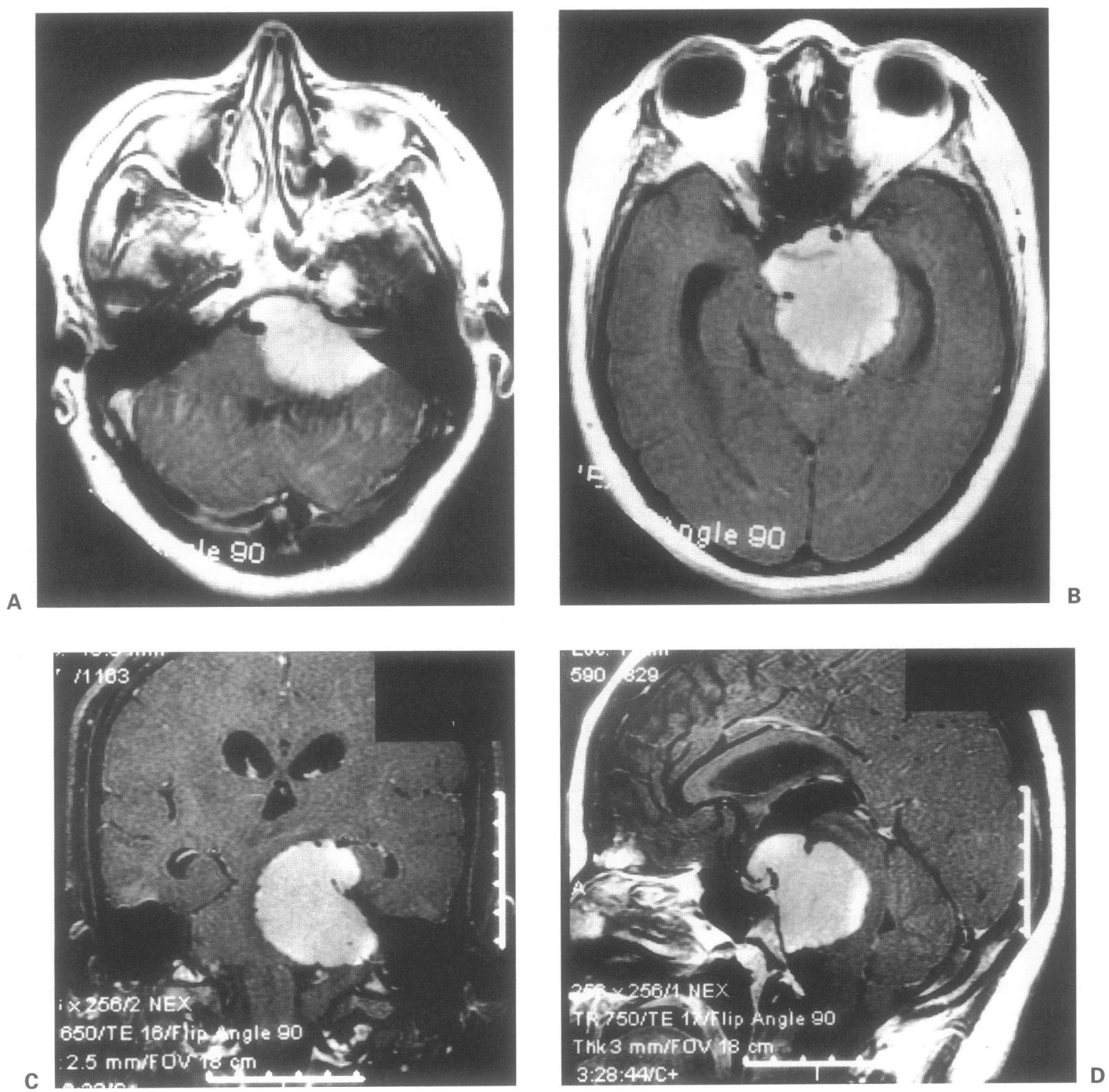

Figure 1 (A and B) Axial (at two different levels), (C) coronal, and (D) sagittal magnetic resonance images of the patient's massive petroclival meningioma.

arachnoidal plane is often present. Microsurgical intra-arachnoidal dissection frees the vessels. The need to establish an intra-arachnoidal plane at the brain stem cannot be overemphasized if this lesion is to be removed safely. Hence, pia mater, perforating arteries, and venous structures on the surface of the brain stem must be preserved.

The extension into the posteroinferior portion of Meckel's cave and the cavernous sinus could also be followed. The plane of dissection is often present against the posterior loop of the carotid artery. The utmost gentleness is required to avoid injuring $\mathrm{CN}$ V. Along with the risk of injuring $\mathrm{CN}$ $\mathrm{V}$ and VII, the incidences for corneal ulceration and later loss of vision are high. Furthermore, manipulating $\mathrm{CN} \mathrm{V}$ might produce the rare but agonizing syndrome of delayed intractable deafferent facial pain. 
The posterior upper clivus bone is involved toward the sphenoid sinus and must also be drilled to achieve grade I removal in this case. By virtue of the total petrosectomy approach, the internal auditory meatus is exposed completely, and the sleeve of tumor around the facial nerve into the internal auditory meatus, which is apparent on the MRI, can be followed.

The surgical goal is safe and complete removal of this tumor. It is the definitive way of achieving the best chance for a cure and for minimizing the chance of a recurrence. If any piece of tumor adheres tightly to the basilar artery, carotid artery, or brain stem, it can be left in place. Additional management with radiosurgery can then be considered or when the residual demonstrates growth on follow-up examination.

\section{POSTOPERATIVE MANAGEMENT}

Complications usually stem from cranial nerve dysfunction. The lower extension risks lower cranial nerve dysfunction, which is associated with the highest morbidity because of the risk of aspiration and pulmonary complications. The combination of CNs VII and V is discussed above. The portion of CN VI just before it enters the petroclival Dorello's canal is the most vulnerable segment. The ventricles may still be enlarged; however, we advise removing the tumor before shunting. If the ventricles enlarge after surgery and the patient develops a full-blown syndrome of hydrocephalus, then a permanent shunt must be placed.

In all skull base surgery, cerebrospinal fluid (CSF) leaks require particular attention in planning the surgical reconstructive closure at the opening. We believe that vascularized tissue is the best means of avoiding a CSF leak; hence, our technique rotates the temporal muscle and temporal fascia. If hydrocephalus ensues, the risk of CSF leakage increases and shunting becomes necessary. Otherwise, we do not use routine spinal drainage in these cases.
Such tumors remain neurosurgical challenges. The risks and rewards associated with each individual treatment are high, but microsurgical and skull base techniques, coupled with meticulous perioperative care, have improved our ability to remove these lesions safely.

$$
\begin{array}{r}
\text { Ossama Al-Mefty, M.D. } \\
\text { Department of Neurosurgery } \\
\text { College of Medicine } \\
\text { University of Arkansas for Medical Sciences } \\
4301 \text { West Markham, Slot } 507 \\
\text { Little Rock, Arkansas 72205-7199 } \\
\text { E-mail: almeftyossama@uams.edu }
\end{array}
$$

Considering the patient's young age, the size of her tumor, and the extent of brain stem compression, surgery is indicated to remove this giant petroclival meningioma.

\section{PREOPERATIVE MANAGEMENT}

$\mathrm{T}_{2}$-weighted magnetic resonance imaging (MRI) is important to determine whether there is a good arachnoid plane between the tumor and the brain stem. It also may provide some idea of the tumor's density. Cerebral angiography is important to evaluate the tumor's vascularity and the vertebrobasilar arteries and its branches. The venous phase especially will show the size and collateralization of the sigmoid sinus and the presence and position of the vein of Labbé. If possible, the tumor would be embolized through external and internal carotid feeders using polyvinyl alcohol particles, preferably 7 to 10 days before the operation. An audiogram should be obtained to determine the extent of the patient's hearing.

\section{SURGICAL MANAGEMENT}

A team approach is planned with a neurosurgeon and neuro-otologist. Neuroanesthesia will include muscle relaxation, and evoked potentials and facial 
nerve function must be monitored. A right frontal ventriculostomy is performed at the beginning of surgery; cerebrospinal fluid (CSF) is drained to relax the brain. I would employ the partial labyrinthectomy, petrous apicectomy, transpetrosal approach. The tumor is debulked with bipolar cauterization, scissors, and suction. The ultrasonic aspirator may be useful.

Key points in the operation include opening Meckel's cave and dissecting cranial nerves (CN) V, VII, VIII, VI, and III, the encased basilar artery, and the anterior inferior cerebellar artery from the brain stem. The brain should be retracted no more than $1 \mathrm{~cm}$ beyond the normal location of the temporal lobe or cerebellum. CN VI, possibly bilaterally, will be encased and can be dissected free. CN IV will be encased and may have to be sacrificed. All other nerves can be preserved. At the end of the procedure, dural grafting, a fat graft, titanium mesh, and artificial bone cement are used for reconstruction. The operation is expected to take about $8 \mathrm{~h}$. Postoperatively, the patient should be able to be extubated and observed in the intensive care unit for 1 to 2 days. The ventriculostomy may be removed 4 to 5 days after surgery. The expected duration of hospitalization is 7 to 8 days, depending on the patient's recovery. I would expect to achieve gross total tumor excision. If small remnants are left, they can be treated with gamma knife radiosurgery.

\section{POSTOPERATIVE MANAGEMENT}

A permanent trochlear nerve palsy and some residual numbness of $\mathrm{CN} \mathrm{V}$ may be expected. Temporary deficits of CN III, VI, and VIII should resolve. In my hands, the risks of death and hemiparesis are extremely low. The patient may require eye muscle surgery if diplopia persists due to abducens nerve paresis.

The patient will undergo MR imaging 3 months after surgery. If residual tumor is present, it will be treated by gamma knife radiosurgery. Fol- low-up imaging is indicated once a year for 5 years and then can be reduced to once every 2 to 3 years.

\author{
Laligam N. Sekhar, M.D. \\ Mid-Atlantic Brain and Spine Institutes \\ 3301 Woodburn Road, Suite 202 \\ Annandale, Virginia 22003 \\ E-mail:1sekhar@aol.com
}

Given the patient's neurologic history and severe brain stem compression, surgical resection of this tumor is indicated. Because the patient is young, I would recommend aggressive resection aimed at complete removal of the tumor.

\section{PREOPERATIVE MANAGEMENT}

Given the surgical approach, a computed tomography (CT) scan of the temporal bone must be obtained to assess the bony anatomy. In this case, a cerebral arteriogram is also needed to reveal the status of the basilar artery, which is encased by the tumor. The vascularity of the tumor will also be apparent; if possible, the tumor should be embolized before the operation. The venous anatomy should be evaluated with respect to the venous drainage of the temporal lobe, the dominance of the transverse sinus, and communication at the torcula. The height of the jugular bulb will influence the caudal extent of the access along the clivus. The $\mathrm{T}_{2}$-weighted MRI scan should be reviewed to determine the presence of pial invasion and brain stem edema. This important prognosticator negatively impacts the separability of the tumor from the brain stem.

In this case, a wide and shallow exposure is important; therefore, most of the left temporal bone is removed given the loss of hearing in that ear. The surgeon can then identify the facial nerve distal to the tumor and also is able to remove the tumor in the internal auditory canal. The wide exposure facilitates dissection of the basilar artery and its branches, which may be intimately involved by the tumor. 
Intraoperative monitoring of somatosensory evoked potentials as well as facial nerve electromyographic (EMG) responses can help improve the safety of the operation and to preserve the facial nerve.

\section{SURGICAL MANAGEMENT}

The operation is performed in conjunction with a neuro-otologist. Because the patient has no hearing in the left ear, the entire temporal bone can be drilled away for a wide approach. A left-sided temporal and posterior fossa approach would be my choice. The patient is positioned supine with the head and neck rotated 60 degrees to the opposite side. Brain relaxation is essential, and a ventricular drain is inserted at the beginning of the operation.

On the left side, a large $\mathrm{C}$-shaped incision, extending from above to behind the ear, is made. The scalp is elevated and the ear canal is sutured closed. The temporalis muscle and the nuchal muscles are elevated. A combined temporal and posterior fossa bone flap is elevated straddling the transverse sinus. The temporal bone, including the labyrinth, is drilled away down to the jugular bulb. The middle ear is obliterated to prevent cerebrospinal fluid (CSF) leak. The facial nerve is unroofed but not transposed.

The temporal dura is opened horizontally, and the undersurface of the brain is inspected for draining veins. The dura in front of the sigmoid sinus is opened vertically from the jugular bulb up to the superior petrosal sinus. This sinus is coagulated and divided. The dura of the temporal floor is divided in front of the temporal veins and the incision continues toward the tentorial incisura, posterior to the entry of the fourth cranial nerve.

The temporal lobe is elevated with the posterior leaf of the tentorium, and the sigmoid sinus and the tentorium are retracted posteriorly. The tumor is debulked progressively while one carefully looks for the cranial nerves. As the cranial nerves are identified and dissected free from the tumor, the base of the tumor is detached from the clivus.
As the tumor is debulked, the capsule of the tumor is separated from the brain stem.

\section{POSTOPERATIVE MANAGEMENT}

During the early postoperative period, paralysis of the facial nerve and the lower cranial nerves is managed carefully. The eye is adequately protected, and speech and swallowing are assessed before enteral feeding is instituted. In the event of lower cranial nerve paralysis, a gastrostomy is performed early to maintain nutrition. Aspiration should also be monitored carefully.

Residual tumor may be managed expectantly or treated early with radiation. If the size of the tumor permits, treatment with stereotactic radiosurgery is preferable. If no irradiation is given, the residual tumor can be observed and treatment can be considered if the tumor grows.

Chandranath Sen, M.D. Department of Neurosurgery St. Luke's-Roosevelt Hospital Center 425 W. 59th Street, Suite 4E New York, New York 10019

E-mail: csen@slrhc.org

A young, healthy woman sought treatment for a myriad of symptoms and signs, the most worrisome related to brain stem compression, such as limb weakness, hypesthesia, and Babinski's sign. The available magnetic resonance images (MRI) showed a petroclival meningioma that involved the clivus in zone I (from dorsum sella to the internal auditory canal (IAC) and zone II (from IAC to jugular foramen). It further extends anteriorly into the sella turcica and posterior cavernous sinus.

\section{SURGICAL MANAGEMENT}

The primary indication for surgery is decompression of the brain stem. Once cytoreduction is achieved, radiotherapy will be used for tumor con- 
trol. En route to achieving these goals, preserving this patient's baseline neurologic function must also be attempted. I believe that a total resection is unachievable and that the attempt would incur serious complication out of proportion to the benefit attained.

Because brain stem compression is my primary objective, the focus of the surgical approach is in the posterior fossa. A presigmoid approach with a posterior petrosectomy will afford access to the tumor in zone II. A labyrinthectomy will be included because the patient is already deaf on the affected side. The facial nerve, however, should not be mobilized from the fallopian canal because the ability of the nerve to recover from this insult is poor, and the added exposure is insufficient to convert a partial resection to a cure. A temporal craniotomy and anterior petrosectomy will gain access to the tumor in zone I. Once again, the cochlea under the geniculate ganglion can be drilled to gain a wider surgical window. With the temporal craniotomy, a subtemporal approach can be used to debulk the portion of the tumor above the dorsum sella. I would not add a frontotemporal craniotomy or orbitozygomatic osteotomy or approach the cavernous sinus tumor. The surgical approach can become so long as to interfere with performance of "the critical portion of the operation." One should be realistic: If residual tumor will be left in the posterior fossa, why threaten the patient to remove a little tumor in the cavernous sinus region?

During resection, protection of the arteries and brain stem will largely determine the ultimate success of the procedure. In the most likely circumstance, a cleavage plane will be found between the basilar artery and tumor. Extreme care must be taken to preserve the small brain stem perforators. There is no apparent subarachnoid plane between the tumor and the brain stem and the brain stem is edematous, implying pial disruption $\left(\mathrm{T}_{2}\right.$-weighted MRI would corroborate this opinion). The tumor will be adherent. Some capsule will probably have to be left behind but enough must be removed to allow the brain stem to reexpand. This is the art and science of the case. The science is in knowing the anatomy, using frameless stereotactic guidance to estimate the degree of resection, and monitoring somatosensory evoked potentials (SSEPs) to predict the likelihood that continuing surgery will cause permanent brain stem dysfunction (anecdotally based on when the amplitude of the SSEP decreases or its latency increases by $50 \%$ ). The art is in knowing when to quit.

This strategy is aimed at stalling for time in the hope that treatments will advance during the next 10 to 20 years. Trust that it will. Write your goals on the board in the operating room. Read them periodically. Don't let this degenerate into a personal battle between you and the tumor. Harry R.van Loveren, M.D.
Mayfield Clinic
3219 Clifton Avenue, \#110
Cincinnati, Ohio 45220
E-mail: hvanloveren@mayfieldclinic.com 